\title{
Two-fluid models for the winds of OB stars
}

\author{
J. H. Guo \\ National Astronomical Observatories/Yunnan Observatory, Chinese Academy of Sciences, \\ P. O. Box 110, Kunming 650011, China. \\ email: guojh@ynao.ac.cn
}

\begin{abstract}
A two-fluid model to OB stars is investigated, in which the flow is described by a set of two components, One for the dense clumps and the other for the smooth gas. The two components are coupled by friction drag. The velocity structure of clumps is assumed to be beta law, thus, the velocity structure of the second component could be attained by shooting method. The result is compared with the X-ray observations.
\end{abstract}

Keywords. Stars: supergiants, stars: early-type, hydrodynamics

\section{Introduction}

Hot stars of spectral O and B have been observed to have X-ray emission. Most of the $\mathrm{X}$-ray emission from early-type stars appears to originate in their winds with temperature of several million degrees (Waldron \& Cassinelli 2007). X-ray emission in normal OB stars is generally explained in terms of shock heating of the supersonic, radiation-driven winds (Lucy \& White 1980; Cohen et al. 2006). Shocks distributed throughout their winds and most likely formed from instabilities of line-driven flow (Lucy \& Solomon 1970).

\section{The model}

For the multiphase hydrodynamical flows, Pistinner \& Shaviv (1993) present a formalism at the concept of spatial averages. The advantages of the technique are that it can formulate the equations in a straightforward physical interpretation. We only present the results, for the details reader can refer to Pistinner \& Shaviv (1993) and Soo (1989). The smooth gas and clumps are coupled by friction. These clumps are accelerated by stellar radiation and slowed down by gravity and friction force with the smooth gas. The smooth gas is accelerated in terms of drag between clumps and gas. Thus we require the equations of mass and momentum conservation.

Dynamical equations for the two-phase flow can be written as

$$
\nabla \cdot\left(\alpha_{g} \rho_{g} v_{g}\right)=\nabla \cdot\left(<\rho_{g}>v_{g}\right)=0
$$

and

$$
\begin{gathered}
\nabla \cdot\left(\alpha_{c} \rho_{c} v_{c}\right)=\nabla \cdot\left(<\rho_{c}>v_{c}\right)=0 . \\
\nabla \cdot\left[<\rho_{g}>v_{g} v_{g}\right]=-\nabla P_{g}+\alpha_{c} \nabla P_{g}+<\rho_{g}>g+\frac{1}{2} C_{D} \frac{<\rho_{C}><\rho_{g}>}{m} U^{2} A \\
\nabla \cdot\left[<\rho_{c}>v_{c} v_{c}\right]=-\alpha_{c} \nabla P_{g}+<\rho_{c}>g-\frac{1}{2} C_{D} \frac{<\rho_{C}><\rho_{g}>}{m} U^{2} A+<\rho_{c}>g^{r a d}
\end{gathered}
$$

In this paper we only consider the motion of gas. The velocity structure of clumps is assumed to be $\beta$ law. Thus we only solve Eqs. (2.1) and (2.3). 


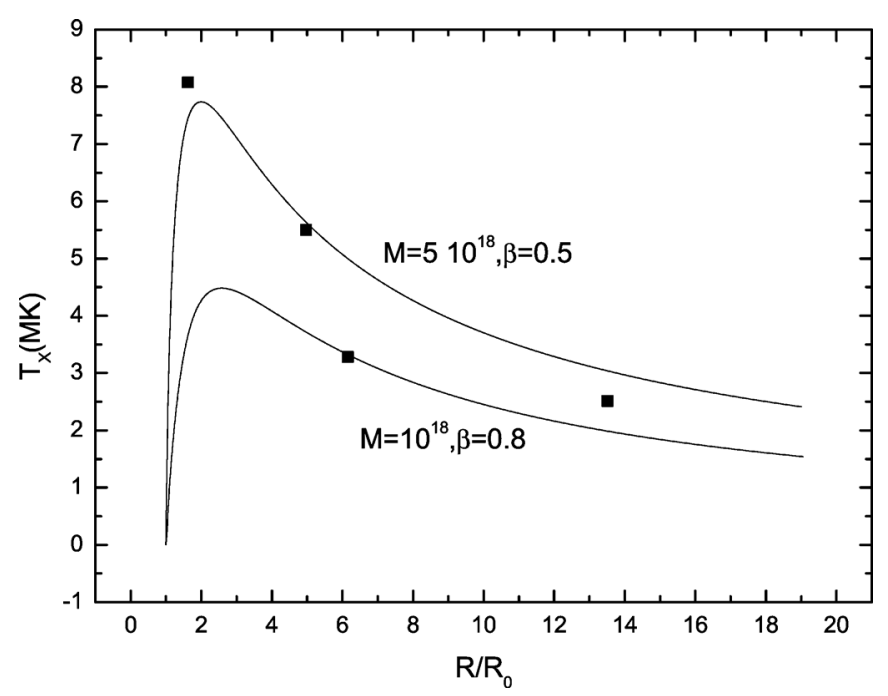

Figure 1. The dependence of $T_{X}$ on the radius.

The solution of the differential Eqs. (2.1) and (2.3) can be obtained by numerical method. The momentum equation has a singularity at the point where $v_{g}=a$. The position of singularity is determined when numerator and denominator go to zero simultaneously. To perform the condition, a suitable lower boundary condition is necessary. We apply shooting method to find the lower boundary condition.

The typical O-type star $\zeta$ Ori A is ideal candidate to test our X-ray model because it have been study in detail (Feldmeier et al. 1997; Waldron \& cassineli 2000). A important test to our model is to compare the X-ray temperature. For the post-shock temperature, we take $T_{s}=1.44 \times 10^{5}\left(\frac{U}{100 K M s^{-1}}\right)^{2} K$.

\section{Results}

The spatial X-ray temperature distributions show that the temperature decreases with the increase of radius. Our model predicted the trend. The result also hints that the mass of clump can not greater than $5 \times 10^{18}$.

\section{Acknowledgements}

This work was supported by the Knowledge innovation Program of Chinese Academy of Sciences.

\section{References}

Cohen D. H. et al. 2006, MNRAS, 368, 1905

Feldmeier A., Puls J., \& Pauldrach W. A. 1997, A\&A, 322, 878

Lucy L. B. \& Solomon P. M. 1970, ApJ, 159, 879

Lucy L. B. \& White R. L. 1980, ApJ, 241, 300

Pistinner S. \& Shaviv G. 1993, 1993, ApJ, 414, 612

Soo S. L. 1989, Particulates and Continuum: Pultiphase Fluid Dynamics (New York:Hemisphere) Waldron W. L. \& Cassinelli J. P. 2000, ApJ, 548, L45

Waldron W. L. \& Cassinelli J. P. 2007, ApJ, 668, 456 